\title{
An Assisted Obstetric Delivery Device for Resource-Limited Settings
}

\author{
John P. Hessburg \\ Department of Biomedical Engineering \\ University of Michigan \\ jackhess@umich.edu \\ Prithvi Murthy \\ Department of Biomedical Engineering \\ University of Michigan \\ pmurth@umich.edu
}

\author{
Daniel P. Ostrowski \\ Department of Mechanical Engineering \\ University of Michigan \\ daniostr@umich.edu \\ Keval D. Patel \\ Department of Mechanical Engineering \\ University of Michigan \\ keval@umich.edu
}

\author{
Kathleen H. Sienko, PhD \\ Departments of Mechanical and Biomedical Engineering \\ sienko@umich.edu
}

\begin{abstract}
Pregnancy-related mortalities account for the death of 350,000 women annually, and $99 \%$ of these mortalities occur in developing countries. Obstructed labor is one of the four leading causes of maternal mortality, in part because of inadequate access to assisted delivery technology. Vacuums and forceps in common use do not meet the functionality required by end-users due to the need for substantial training and unreliable energy sources (electricity is not always available in rural clinics), and risks or complications can occur if the devices are used improperly. An assisted obstetric delivery prototype was designed for use in resource-limited settings based upon the input gathered during 15 weeks of clinical observation and interaction with Ghanaian health care workers in summer 2011. The manually operated prototype consists of a pronged applicator and an extractor sleeve. Preliminary evaluation of the prototype has been completed, and future work will involve redesigning the extractor's securing mechanism, modifying the applicator's shape to improve deflection characteristics, and conducting validation studies in human subjects.
\end{abstract}

Index Terms - Project-based learning, clinical immersion, ethnographic investigations, assisted delivery, global health design, maternal health, obstructed labor

\section{INTRODUCTION}

Approximately 350,000 women die annually as a result of complications related to pregnancy. ${ }^{1}$ Ninety-nine percent of these deaths occur in developing countries. ${ }^{2}$ Yet, many current intervention and treatment options remain unavailable to a substantial portion of the world's population., ${ }^{2,3}$ The United Nations Millennium Development Goal 5 was announced in 1990 with the objective of reducing the maternal mortality ratio by $75 \%$ by $2015 .^{4}$ To date, the annual decrease in maternal mortality is $2.3 \%$, far short of the $5.5 \%$ average annual decrease necessary to meet the UN's goal. ${ }^{2}$

Obstructed labor, one of the four leading causes of maternal mortality ${ }^{5}$, is defined as an inability of the baby to progress through the birth canal despite strong uterine contractions. This 
condition accounts for 4.1-13.4\% of maternal deaths in Africa, Asia, South America, and the Caribbean ${ }^{6}$, or approximately 42,000 deaths per year. ${ }^{7}$ The lengthy application of pressure by the fetal head on the vaginal wall as a result of obstructed labor can cause a fistula, or abnormal connection, between the vagina and the rectum or bladder. Available treatment options for obstructed labor include cesarean section and assisted vaginal delivery, the latter through forceps or vacuum extraction. These methods can be effective with soft tissue obstructions, during a prolonged second stage of labor, when maternal effort is lacking, or to expedite delivery in the event of fetal distress. 7,8

In the developing world, assisted delivery is the least-available obstetric intervention. ${ }^{9}$ Since the use of forceps requires significant training ${ }^{14}$, obstetricians generally are the primary users of this device. Vacuum extraction is promoted as an alternative because the procedure can be used by a range of health providers, and it is associated with less trauma to both mother and infant. ${ }^{9,10}$ However, the vacuums available in resource-limited settings are often outdated, their functionality is limited due to leaking tubes and broken bottles, or the supply of electricity is unreliable. ${ }^{9}$ Thus, several researchers ${ }^{9-12}$ as well as the Bellagio workshop of maternal health experts $^{13}$ have recommended the development of assisted delivery devices appropriate for resource-limited settings.

The aim of this study was to conduct a needs assessment in Ghana on the topic of maternal health using ethnographic investigation techniques, co-define a design project with Ghanaian clinicians, co-create a novel design to mitigate the specific maternal health problem, and perform a preliminary evaluation of the prototype.

\section{ASSISTED DELIVERY DEVICES}

Forceps, the most versatile instruments used for assisted delivery (Figure 1), generally consist of two steel blades and a handle that attaches the blades to one another. The blades are inserted into the vagina and then closed around the presenting part of the baby. Compared to other assisted delivery devices, forceps can deliver both breech and cephalic presentations, and also can rotate the baby for easier delivery. The drawback is that the force applied can cause higher rates of maternal vaginal and perineal trauma, and neotnatal facial trauma. ${ }^{14}$

In vacuum extraction a cup attaches to the fetal scalp and a vacuum then applies traction to deliver the baby. Vacuums are designed to intentionally fail by detaching from the fetal scalp whenever an unsafe pressure is reached. There are two categories of vacuum extractor in use: metal or rigid cups, and deformable cups. The metal cup forms a stronger seal with the baby's scalp, and is less likely to detach. The drawback is that the metal cup can cause extensive soft tissue damage to the fetal scalp when the vacuum is applied. ${ }^{14}$ Deformable cups (Figure 1), generally made from polyethylene or silicone, do not establish as tight a seal and are more likely to detach. ${ }^{14}$ However, their deformable nature causes less damage to the fetal scalp. ${ }^{14}$ Some devices attach to electronically powered suction machines, while others use a hand or foot pump to generate pressure.

Clinical Innovations's Kiwi Vacuum Delivery System (Figure 2) is a disposable hand-held pump with a hard plastic cup and a foam insert to prevent excessive caput formation. A newer model, the Vacca Reusable OmniCup, has a trap to prevent fluid from being drawn into the pumping mechanism. The cup and fluid trap are sterilizable, so the device can be used multiple times. 

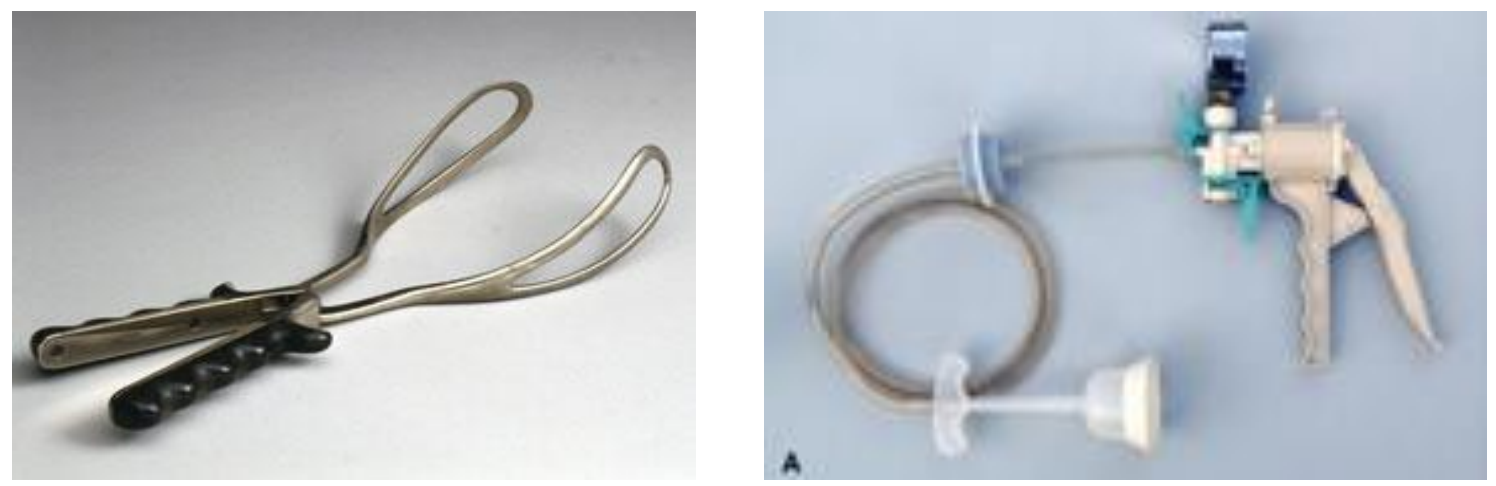

FIGURE 1

SIMPSON FORCEPS $^{15}$ (LEFT) AND MITYVAC VACUUM DEVICE ${ }^{16}$ (RIGHT)

The Odon device (Figure 2), developed explicitly for resource-limited settings, is undergoing clinical trials in Argentina and South Africa. It has a disposable, folded sleeve of polyethylene that is applied to the head of the baby with a reusable bell-shaped inserter. A small amount of air is pumped into the inner sleeve, the inserter is removed, the sliding effect of the two sleeves creates an air lock, and traction is then applied to deliver the baby.
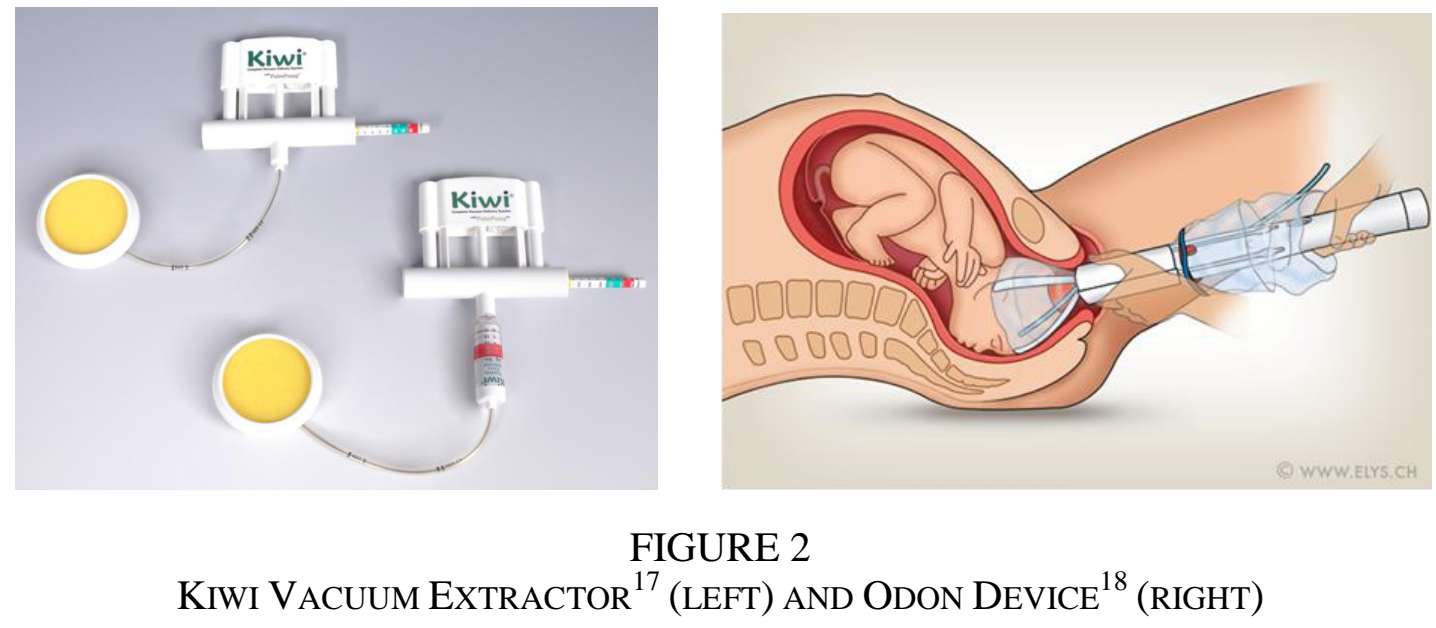

\section{METHODS}

The needs assessment conducted in Ghana identified a need for an inexpensive assisted delivery device that applies less pressure to the baby's head, is easier to use, requires less training than forceps, the Kiwi device, or the Odon device, and does not depend on electricity.

A common problem associated with designing devices intended for use in resource-limited settings is the failure to account for the desires and needs of the end-user, instead focusing on what the designer thinks the end-user needs. ${ }^{13}$ The University of Michigan Global Health Design specialization provides engineering students with an opportunity to address global health challenges through clinical immersion experiences and capstone design. The program emphasizes co-creation as part of the comprehensive design process, including experiential learning, intercultural training, and in-depth exposure to a specific global health challenge. 
Students have the option of completing either one or two semesters of design following their clinical immersion. Those opting to complete two semesters of design typically enroll in the Multidisciplinary Design Minor Global Health Design (GHD) specialization. This specialization is a rigorous, two-semester design course which features project scoping/clinical immersion in domestic or international clinics, co-creation with the target community, technology introduction, and re-design.

Three of the four student authors participated in a clinical immersion experience in Ghana during the summer of 2011. In order to create user-centric design, team members conducted clinical observations and interviews to identify challenges related to maternal health care. The health care providers interviewed were obstetricians, midwives, nurses, and community health workers from primary, secondary, and tertiary level health care facilities. From the list of challenges derived from these interviews and observations, input from experts both in Ghana and at the University of Michigan identified assisted delivery as a high priority.

\section{User Requirements}

Interviews with potential end-users and a survey distributed to obstetricians and midwives at the University of Michigan and in Ghana and also distributed via several online obstetrics and midwifery forums were used to determine the most desirable requirements for an assisted delivery device. In order of priority, the requirements (Table 1) identified were: safety of the mother, safety of the end-user, manually powered by the end-user, easy for one person to use, and affordable.

\section{Engineering Specifications}

The end-user requirements were translated into engineering specifications (Table 1) through literature research and expert input.

\section{DESCRIPTION OF DESIGN}

The design has two components: an applicator and a fabric extractor. The extractor is loaded onto the applicator, which is then used to apply the elastic fabric around the baby's head. The fabric is secured, the applicator is removed, and traction is applied to the end of the fabric sleeve to deliver the baby. Applying traction distributes pressure around the face of the baby, rather than along the chin and the base of the head, as with the Odon device, or the sides of the face, as with forceps.

The applicator has three separate elements: a three-pronged compliant plastic unit, a cone, and a plunger handle. The prongs, cone, and plunger handle will be made of the polymer polyethylene terephthalate (PET), which is compliant, resilient, biocompatible, and can be massproduced through injection molding. The plunger is inserted into the pronged component, and the two units slide relative to one another. 
TABLE 1 USER REQUIREMENTS AND ENGINEERING SPECIFICATIONS

\begin{tabular}{|c|c|c|}
\hline Rank & User Requirement & Engineering Specifications \\
\hline 1 & Safe for the mother & $\begin{array}{l}\text { Tensile stress exerted on maternal tissue } \leq 11.72 \\
\mathrm{MPa}^{19} \\
\text { Maternal tissue between device and baby }=0 \mathrm{~cm}^{3 *} \\
\text { Perineum supported by one hand }{ }^{*} \text { when delivering } \\
\text { head } \\
\text { Non-toxic: average MP from SMI assay } \leq 7 \%{ }^{20} \\
\text { Edge radius of curvature } \geq 2 \mathrm{~mm}\end{array}$ \\
\hline 2 & Safe for the baby & $\begin{array}{l}\text { Release device in } \leq 30 \text { seconds }^{21} \\
\text { Traction force applied to baby } \leq 184 \mathrm{~N} \\
\text { Compressive pressure on baby's head } \leq 128.2 \mathrm{kPa}^{22} \\
\text { Device applied for } \leq 15 \text { minutes } \\
\text { Non-toxic: average MP from SMI assay }<7 \%{ }^{20} \\
\text { Edge radius of curvature } \geq 2 \mathrm{~mm}\end{array}$ \\
\hline 3 & Powered by user & $100 \%$ of energy supplied by end-user ${ }^{*}$ \\
\hline 4 & Easily operated by one user & $\begin{array}{l}\text { End-user training time } \leq 1 \text { hour to achieve } \geq \text { score of } \\
770 \text { on simulator testing } \\
\text { Pulling force applied by end-user } \leq 148.2 \mathrm{~N}^{23} \\
\text { Baby deliverable with } \leq 2 \text { hands } \\
\text { Device applied with } \leq 2 \text { hands } \\
\text { Weight } \leq 4 \mathrm{Kg}^{*}\end{array}$ \\
\hline 5 & Affordable & Procedure cost $\leq \$ 37$ US \\
\hline
\end{tabular}

*: End-user derived specification

A prototype was developed to demonstrate proof of concept during the fall 2011 and winter 2012 semesters. (Figures 3 and 4) The extractor is made of 86\% nylon, 14\% spandex fabric, from a pair of commercially available tights/stockings. The applicator prongs slip into three pockets sewn inside the extractor that secure the applicator while it is being applied over the baby's head. A cord runs through a seam along the end of the sleeve to form a drawstring, and then through a cord lock attached to the fabric. Pulling the cord towards the end-user tightens the drawstring; two knots on the drawstring prevent it from closing too tightly. The drawstring is used to secure the extractor to the baby's chin as traction is initially applied, and prevents the fabric from sliding off of the baby's head.

The cord lock, which prevents movement of the cord in the opposite direction, fixes the diameter of the extractor. A manual release pull disengages the cord lock, allowing the cord to move freely so that the extractor can be removed at any point during the procedure and after successful delivery. The drawstring cords and the release cord run through three loops of fabric along the outside of the sleeve to prevent the cords from tangling or obstructing access. 

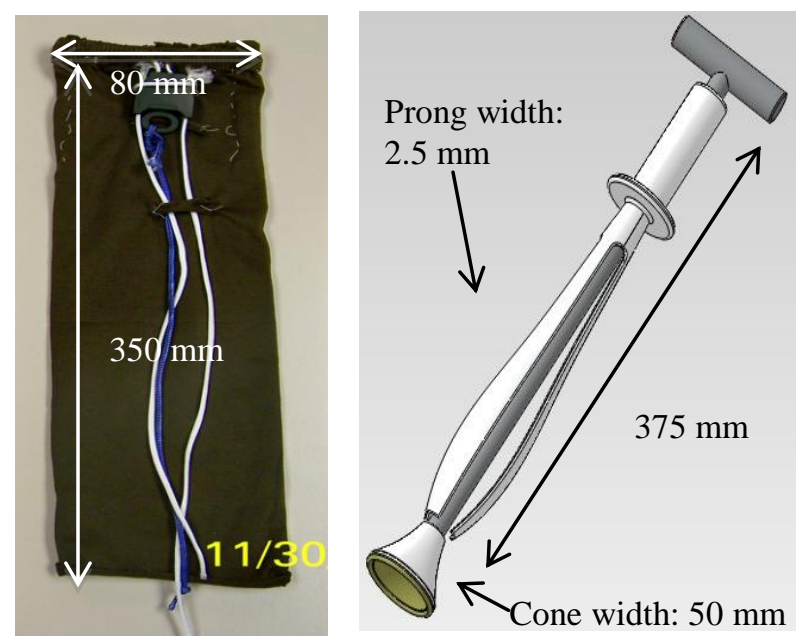

FIGURE 3

EXTRACTOR (LEFT) AND APPLICATOR (RIGHT)

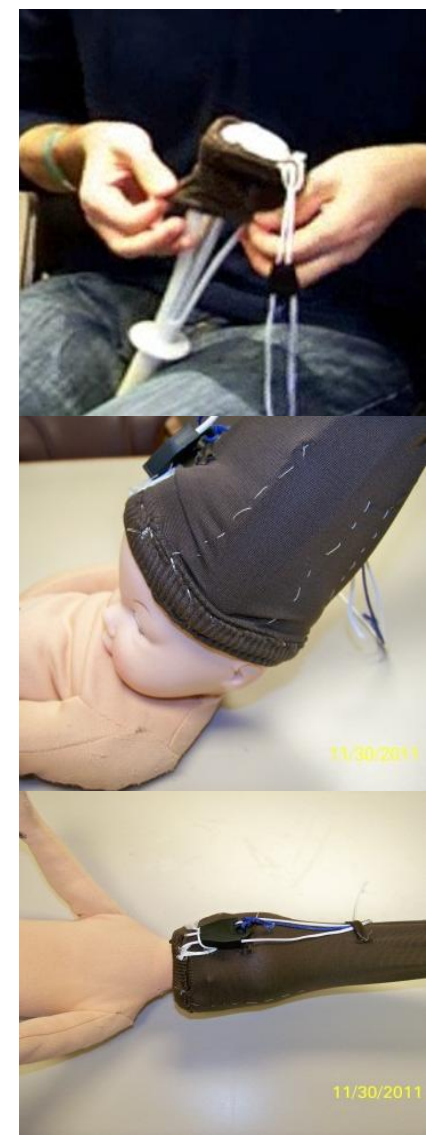

1. Slide fabric extractor over applicator, guiding prongs into sleeves along inside of extractor

3. Slide handle of applicator along shaft while keeping cone on baby's head

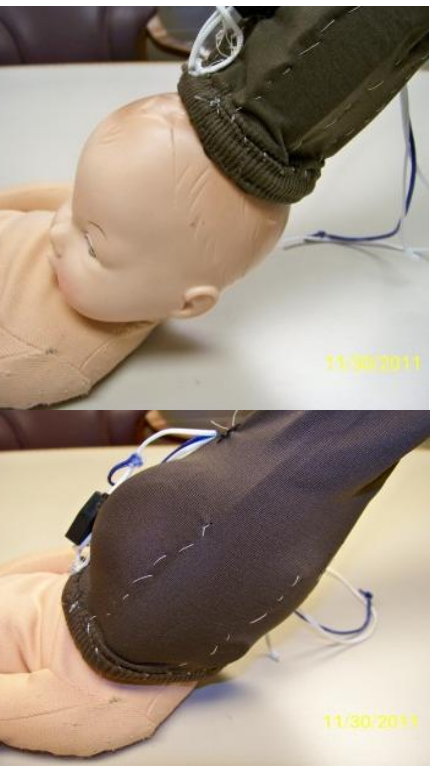

2. Place cone on baby's head

\section{Apply traction to} deliver baby
FIGURE 4

PROTOTYPE APPLICATION
4. Secure cord lock by tightening drawstring, remove applicator 


\section{VALIDATION AND EVALUATION}

To evaluate the compressive pressure applied to the baby's head, a Tekscan FlexiForce sensor was attached to a model fetal head. The compressive pressure was measured at the level of the drawstring and at the point of widest diameter, i.e. the locations where the greatest amount of force was expected. Prototypes fabricated from three fabric blends were tested by affixing the force sensor to the head, applying and securing the extractor, and pulling at $160 \mathrm{~N}$, the upper limit of traction force. The results are shown in Table 2.

TABLE 2

COMPRESSIVE PRESSURE ON SIMULATED FETAL HEAD (KPA)

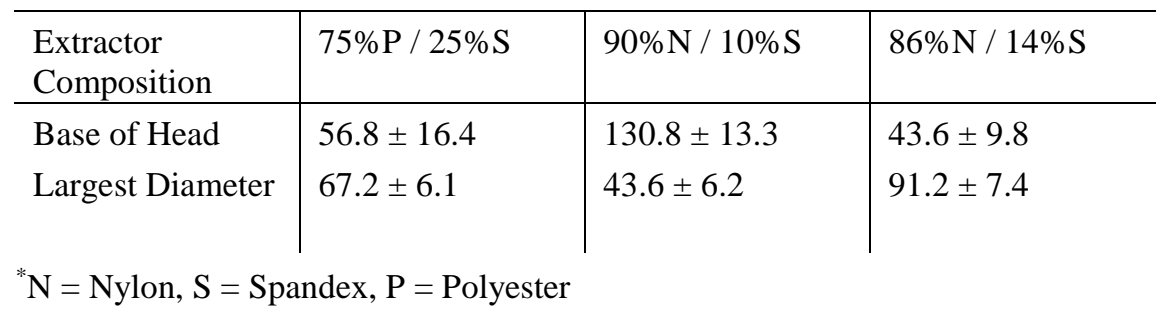

The experimentally determined force data demonstrated that the compressive pressure was not concentrated solely at the base or the largest diameter of the model head. With the exception of the pressure at the base of the head in the $90 \%$ nylon/10\% spandex blend, all pressures were below the maximum safe pressure of $128.2 \mathrm{kPa}$. The material was chosen to demonstrate the ability of commercially available fabric to be a safe material for the extractor, and demonstrated the prototype's (the $86 \%$ nylon/14\% spandex blend) ability to apply a safe level of compressive pressure to the head of the baby at the two areas of greatest force.

The material of the fabric is designed to fail when a high traction force is applied. Tensile testing of the fabric used in the extractor yielded an average failure force (defined as the point at which the material begins to rip) of $170.0 \pm 8.9 \mathrm{~N}$. This failure force is slightly below the safe traction force that can be applied to the baby. This safe level of force was derived from currently existing vacuum devices, which are designed to automatically disengage once this force is reached. This level of force is significantly lower than the forces routinely reached by forceps delivery.

The application procedure involves sliding an object between the neonatal head and the vaginal wall. To reduce the risk of tearing or scratching either surface, the applicator has a minimum radius of curvature of $2 \mathrm{~mm}$, which is the minimum radius of curvature of the Simpsons forceps, a commonly used instrument that is inserted in a similar fashion.

To validate the time required to remove the extraction component post-delivery, ten individuals were tested under a specified protocol. These individuals had no familiarity with obstetrics or the operation of the prototype. This was done intentionally to demonstrate that an untrained individual would be able to properly remove the device in a short amount of time. The test results showed that the users removed the device, on average, in 4.38 seconds.

The functionality of the prototype was tested on a Gaumard NOELLE S500 Advanced Childbirth Simulator. (Figure 5) This simulator consists of a model pelvis and uterus with interchangeable vulval inserts and a model baby. The birth canal and vaginal opening are made 
of a rubber material lubricated with talcum powder to provide some friction during delivery. The model baby is placed in the simulated pelvic cavity and pulled through the birth canal.

Several engineering specifications were also evaluated on the simulator: the ability to support the perineum while delivering the baby, the ability of a single end-user to apply the prototype and deliver the baby, and the ability to release the prototype at any point during delivery. Traction was applied to the extractor with one hand, while the other hand remained free to support the perineum. No user needed assistance to apply the prototype and deliver the baby, which demonstrates the possibility for a single end-user to perform the simulated assisted delivery and to meet the design specification of delivery using both hands. The release cord attached to the cord lock was able to unlock the drawstring, which enabled the extractor to be removed both while the baby was in the birth canal and after the baby was delivered.

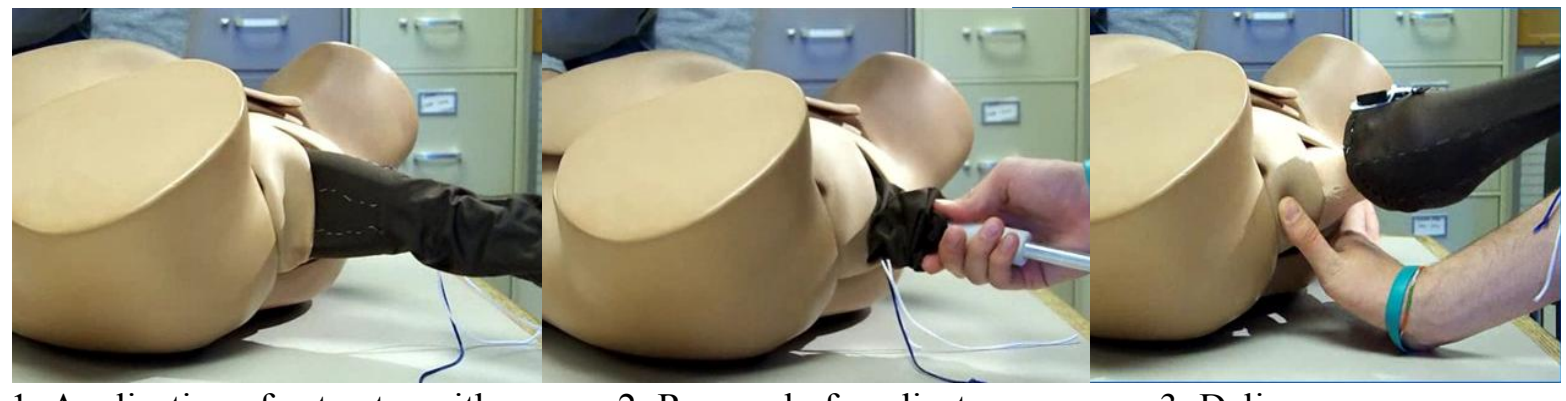

1: Application of extractor with supported perineum

2: Removal of applicator

3: Delivery

FIGURE 5

EVALUATION OF PROTOTYPE ON NOELLE BIRTHING SIMULATOR

The ease of use requirement was evaluated by investigating the time necessary to train endusers in proficient use of the prototype. A training workshop and a simulator testing protocol were developed based on an existing model, ${ }^{24,25}$ and with input from medical training time specialists at the University of Michigan.

Five students were tested before and after participation in the half-hour training workshop. The scoring was based on the time taken to deliver the model, with penalties incurred for errors during the procedure. A higher score (the maximum score was 900 points) indicated a more proficient use of the prototype. The pre-training score was $481.8 \pm 99.1$ and the post-training score was 712.4 \pm 31.0 . The average group member score was $770.3 \pm 20.3$.

The training results show a marked improvement in scores as a result of the training workshop. Four of the five students failed to deliver the simulated baby during the pre-training test, largely because of confusion over proper extractor application. After the training workshop, all five students successfully delivered the simulated baby. The scores for the group after a single half-hour training session were close to the reference scores of the group members, which suggest that end-users can be trained to an appropriate level of proficiency in less than one hour of training.

This design incorporates a reusable pronged applicator and a single-use fabric extractor, so the procedural cost of the design is a function of the applicator cost, applicator lifetime, and extractor cost. Granta Cambridge Engineering Selector (CES) software was used to estimate the mass production cost of the PET component of the applicator $(\$ 2.21)$ and the aluminum handle 
and guide shaft (\$3.66) for a total applicator cost of \$5.87. This estimate considered manufacturing processes, material costs, labor rate, overhead, and economies of scale. The estimated extractor cost of $\$ 2.65$ considers material costs, labor rate, and the cost of sterilization.

The fatigue life of the applicator prongs was used to estimate the lifetime of the applicator. A PET fatigue chart was used to determine the cycle life of the PET prongs according to the loading scenario. The failure was evaluated to be the point at which $10 \%$ of the prongs failed. This scenario occurred at a lifetime of 3000 uses. For a conservative estimate the lifetime was reduced by a safety factor of 1.5 , which yielded a lifetime of 2000 uses. The estimated cost per use of the applicator was then $\$ 5.87$ divided by 2000 , or less than one cent. Thus, the estimated cost per use of the design is $\$ 2.65$. The total estimated procedural cost in a resource-limited setting was determined by adding the cost per use of the extractor and the applicator to the average cost of a normal vaginal delivery, which is $\$ 14.60$ in Ghana. ${ }^{26}$ This yielded an overall procedural cost of $\$ 17.25$ for the design.

As mentioned, team members consulted obstetricians and midwives at the University of Michigan and in Ghana to ensure the safety of the prototype for both the mother and the baby during delivery. In a survey distributed to fifteen obstetricians at the University of Michigan and in Ghana, $80 \%$ agreed or strongly agreed with the statement that the design is safe for the mother, and $47 \%$ that the design is safe for the baby. One respondent disagreed with each statement. One respondent stated "I gave neutral answers as to safety and reduction of MMR - a major limitation is that it has never been tested in an actual delivery." Another responded that "clinical trials must be done before it can be deemed safe."

During a return visit to Ghana in February 2012 end-users provided feedback on the practicality and potential use of the design. Design team members held interactive presentations at two large teaching hospitals to explain the prototype and to gather feedback. Focus groups held with senior obstetricians and midwives gathered additional feedback.

A survey distributed to those attending the presentations yielded 66 responses. Of the midwives and obstetricians who responded, 56.3\% said "yes" and 42.2\% said "maybe" when asked if they thought the design was safe for the mother, and $23.4 \%$ said "yes" and $70.3 \%$ said "maybe" when asked if they thought the design was safe for the baby. Explanations for neutral responses included: "Hands on real babies would be a crucial step in promoting this device." "I would need to do some more practices [sic] to give more comments." "There is the need to use this device and evaluate how effective it will be." When asked if the prototype was easy to assemble, $78.1 \%$ said "yes." An initial concern was that the prototype's appearance could deter midwives or obstetricians from using it; when asked if the prototype appeared frightening, $64.1 \%$ said "no," and $17.2 \%$ said "maybe."

The feedback and preliminary validation results will be used to iterate the design. In fact, bringing the prototype back to the same locations where the initial observations and interviews were conducted allowed further collaboration with the obstetricians and midwives, and strengthened the foundation for future design groups.

\section{CONCLUSION}

The design concept employs a novel technique to address a significant medical challenge in resource-limited settings. Important benefits of this design include the exertion of less pressure on the baby's head and a lower surface pressure than existing forceps and vacuum devices. The 
design is inexpensive to manufacture and to purchase, and the disposable component can be fabricated from locally available materials. The applicator is reusable and can be disinfected with bleach. The prototype itself is independent of external energy sources and simple to use. Enduser training can be accomplished in a short period of time, and the device is not limited to physician use.

Several design issues remain to be addressed. The commercial cord lock used to anchor the extractor is unreliable, bulky, and has a hard, sharp exterior that is not suited for use inside the birth canal. A new method of attachment involving a unidirectional rubber cone lock was developed to secure the design in conjunction with a separate emergency release knot. Separating the two components made the design more robust, decreased the likelihood of failure, and was more user-friendly, although the internal locking mechanism still is being refined for better functionality. Finite element analysis is being conducted using Altair's Optistruct software to optimize the geometry of the applicator. The objective is to minimize the pressure exerted on the baby's head during application. The software can then modify the shape of the applicator based on its material properties.

Although the initial cohort responsible for designing the prototype has graduated, the remaining team members are exploring partnerships with a local global health start-up. Discussions with licensing specialists are also underway. Although substantial human subject testing is required to validate the safety and efficacy of the design, the results obtained to date suggest that if further developed, the proposed device could offer a safe, easy to use, and costeffective solution to obstructed labor.

\section{ACKNOWLEDGMent}

The authors acknowledge the Multidisciplinary Design Minor: Specialization in Global Health Design, the National Collegiate Inventors and Innovators Alliance, the College of Engineering, the International Programs in Engineering, the African Studies Center, the Center for Entrepreneurship, the Center for Global Health, the Clinical Simulation Center, Mr. Harvey Bell, Mr. Amir Sabet, Dr. Aileen Huang-Saad, Mr. Moses Lee, Dr. Thomas Konney, Dr. Tawiah Odoi, Dr. Samuel Obed, Mr. Dominic Anaseba, Dr. Frank Anderson, Dr. Timothy Johnson, Mr. Bijoy Paul, and the clinicians and staff at Komfo Anokye Teaching Hospital, Korle Bu Teaching Hospital, Navrongo Health Research Center, and the University of Michigan Health System.

\section{REFERENCES}

${ }^{1}$ Hogan MC, Foreman K,J., Naghavi M, Ahn S,Y., Wang M, Makela S,M., Lopez A,D., Lozano R, Murray CJL. Maternal mortality for 181 countries, 1980-2008: A systematic analysis of progress towards millennium development goal 5. The Lancet 2010.

${ }^{2}$ WHO | Maternal mortality [Internet].

${ }^{3}$ Callister L.C., Edwards J,E. Achieving millennium development goal 5, the improvement of maternal health. Journal of Obstetric, Gynecologic, \& Neonatal Nursing 2010; 39(5):590-9.

${ }^{4}$ World Health Organization "World Health Report 2005-make every mother and child count". World Health Organization, Geneva (2005), pp. 190-191.

${ }^{5}$ Dolea C, AbouZahr C. Global burden of obstructed labour in the year 2000. Geneva: World Health Organization 2000. 
${ }^{6}$ Khalid S Khan, Daniel Wojdyla, Lale Say, A Metin Gulmezoglu, Paul F A Van Look. WHO analysis of causes of maternal death: A systematic review. Lancet 2007;367:1074.

${ }^{7}$ Bailey PE. The disappearing art of instrumental delivery: Time to reverse the trend. International Journal of Gynecology \& Obstetrics 2005;91(1):89-96.

${ }^{8}$ O’Grady JP, Gimovsky ML, McIhargie CJ. Vacuum extraction in modern obstetric practice. New York: The Parthenon Publishing Group; 1995.

${ }^{9}$ Ameh CA, Weeks AD. The role of instrumental vaginal delivery in low resource settings. BJOG: An International Journal of Obstetrics \& Gynaecology 2009; 116:22-5.

${ }^{10}$ Tsu V,D. REVIEW: Appropriate technology to prevent maternal mortality: Current research requirements. BJOG: An International Journal of Obstetrics \& Gynaecology 2005;112(9):1213-8.

${ }^{11}$ Fauveau V, De Bernis L. Good obstetrics $\square$ revisited: Too many evidence-based practices and devices are not used. International Journal of Gynecology and Obstetrics 2006;94(2):179-84.

${ }^{12}$ Bailey P, Paxton A, Lobis S, Fry D. The availability of life-saving obstetric services in developing countries: An in-depth look at the signal functions for emergency obstetric care. International Journal of Gynecology \& Obstetrics 2006;93(3):285-91.

${ }^{13}$ Tsu VD, Shane B. New and underutilized technologies to reduce maternal mortality: Call to action from a Bellagio workshop. International Journal of Gynecology \& Obstetrics, 2004.

${ }^{14}$ O’Mahoney, F., GJ Hofmeyr, and V. Menon. “Choice of Instruments for Assisted Vaginal Delivery.” WHO. N.p., 10 Nov. 2010. Web.

15 "Simpson Type Obstetrical Forceps." Science Museum. 20 Sept. 2011. Web. <http://www.sciencemuseum.org.uk/objects/obstetrics_gynaecology_and_contraception/A500190.aspx>.

16 “Vacuum Delivery System.” Access Medicine. Web. 20 Sept. 2011. <www.accessmedicine.ca〉.

17 "Clinical Innovations - Kiwi Vacuum Delivery System." Clinical Innovations - Kiwi Vacuum Delivery System. Web. 20 Sept. 2011. <http://www.clinicalinnovations.com/kiwi.htm>.

18 “A New, Simple, Low Cost Instrument for Assisted Vaginal Delivery." Odon Device. Web. 20 Sept. 2011. <http://www.odondevice.org/>.

19 Veronda D., and R. Westmann. Mechanical characterization of skin: Finite deformations. J Biomech 1970;3(1):111-22.

${ }^{20}$ Adriaens, Els. "The Slug Mucosal Irritation Assay: an Alternative Assay for Local Tolerance Testing." National Center for the Replacement, Refinement, and Reduction of Animals in Research (2006): 1-9.

${ }^{21}$ Aldo Vacca. Handbook of vacuum extraction in obstetric practice. London, Melbourne, Auckland: Edward Arnold; 1992.

${ }^{22}$ Pearse, WH. "Electronic Recording of Forceps Delivery. ” American Journal of Obstetrics and Gynecology 1.86 (1963):43-51. Web.

${ }^{23}$ Cheng, Te S., and Tzu H. Lee. "Human Pulling Strengths in Different Conditions of Exertion." Perceptual and Motor Skills (2004): 542-50. Print. 
International Journal for Service Learning in Engineering Vol. 7, No. 2, pp. 1-12, Fall 2012

ISSN 1555-9033

${ }^{24}$ Korndorffer, J. R. "Developing and Testing Competency Levels for Laparoscopic Skills Training. ” Archives of Surgery 140.1 (2005): 80-84. Print.

25 Korndorffer, J., J. Dunne, R. Sierra, D. Stefanidis, C. Touchard, and D. Scott. "Simulator Training for Laparoscopic Suturing Using Performance Goals Translates to the Operating Room." Journal of the American College of Surgeons 201.1 (2005): 23-29. Print.

26 Borghi J, Acquah C, Ekamian G, Filippi V, Ronsmans C, Brugh R, Browne E, Alihounou E. Costs of near-miss obstetric complications for women and their families in Benin and Ghana. Health Policy and Planning. 2003, 18(4): 383-390. 